\title{
Corrigendum: Safety Profiles of Tripterygium wilfordii Hook F: A Systematic Review and Meta-Analysis
}

\author{
Chi Zhang ${ }^{1,2}$, Ping-Ping Sun ${ }^{3}$, Hong-Tao Guo ${ }^{4}$, Yan Liu ${ }^{1}$, Jian $\mathrm{Li}^{3}$, Xiao-Juan $\mathrm{He}^{1,2}$ and \\ Ai-Ping $\mathrm{Lu}^{2 *}$ \\ ${ }^{1}$ Institute of Basic Research in Clinical Medicine, China Academy of Chinese Medical Sciences, Beijing, China, ${ }^{2}$ School of \\ Chinese Medicine, Hong Kong Baptist University, Kowloon, Hong Kong, ${ }^{3}$ School of Basic Medical Sciences, Beijing \\ University of Chinese Medicine, Beijing, China, ${ }^{4}$ The First Affiliated Hospital of Henan University of Traditional Chinese \\ Medicine, Zhengzhou, China
}

Keywords: Tripterygium wilfordii Hook F, safety, adverse event, systematic review, meta-analysis

\section{A corrigendum on}

\section{OPEN ACCESS}

Edited and reviewed by: Adolfo Andrade-Cetto,

National Autonomous University of Mexico, Mexico

*Correspondence: Ai-Ping Lu aipinglu@hkbu.edu.hk

Specialty section: This article was submitted to

Ethnopharmacology,

a section of the journal

Frontiers in Pharmacology

Received: 12 January 2017

Accepted: 30 January 2017

Published: 15 February 2017

Citation:

Zhang C, Sun P-P, Guo H-T, Liu Y,

Li J, He X-J and Lu A-P (2017)

Corrigendum: Safety Profiles of

Tripterygium wilfordii Hook F:

A Systematic Review and Meta-Analysis.

Front. Pharmacol. 8:59.

doi: 10.3389/fphar.2017.00059
Safety Profiles of Tripterygium wilfordii Hook F: A Systematic Review and Meta-Analysis by Zhang, C., Sun, P.-P., Guo, H.-T., Liu, Y., Li, J., He, X.-J., et al. (2016). Front. Pharmacol. 7:402. doi: $10.3389 /$ fphar.2016.00402

In the original article, there was an error. "Aspirin" was referred to instead of "TwHF."

A correction has been made to the last sentence of the Results section in the abstract:

We screened 4137 abstracts for eligibility and included 594 studies in the analysis. The overall incidence of AEs was 26.7\% (95\% CI 24.8\%, 28.8\%) in 23,256 TwHF users. The estimates did vary markedly when stratified by specific study types. The incidence of gastrointestinal symptoms, adverse reproductive outcomes, adverse skin reactions, hematologic events and cardiovascular events were 13.3\% (95\% CI 11.9\%, 14.9\%), 11.7\% (95\% CI 10.3\%, 13.3\%), 7.8\% (95\% CI 6.3-9.5\%), $6.5 \%$ (95\% CI 5.7-7.4 \%) and 4.9\% (95\% CI 1.6\%, $14.3 \%$ ), respectively. The prevalence of irregular menstruation (IM) was increased in patients taking TwHF compared with those given control (odds ratio [OR] 4.65, 95\% CI 3.08 to 7.03). TwHF use has lower risk of weight gain (OR 0.12 [95\% CI 0.04 to 0.39 ]) and hair loss (OR 0.37 [95\% CI 0.18 to 0.78]). Furthermore, long-term TwHF use (>6 months) has a higher AEs incidence (31.0\% [95\% CI 24.5\%-38.5\%]).

The authors apologize for this error and state that this does not change the scientific conclusions of the article in any way.

Conflict of Interest Statement: The authors declare that the research was conducted in the absence of any commercial or financial relationships that could be construed as a potential conflict of interest.

Copyright () 2017 Zhang, Sun, Guo, Liu, Li, He and Lu. This is an open-access article distributed under the terms of the Creative Commons Attribution License (CC BY). The use, distribution or reproduction in other forums is permitted, provided the original author(s) or licensor are credited and that the original publication in this journal is cited, in accordance with accepted academic practice. No use, distribution or reproduction is permitted which does not comply with these terms. 